\title{
锰族长竞选
}

张润弘 ${ }^{\S}$, 张来英 ${ }^{*}$, 朱亚先 ${ }^{*}$

厦门大学化学化工学院, 福建厦门 361005

摘要: 锰在人类的生活和生产中都发挥着重要的作用。本文通过拟人化的手法, 介绍了锰资源开发、锰的性质和应 用, 让读者以生动活泼的方式了解锰及其化合物。

关键词: 锰; 性质; 应用

中图分类号: G64; O6

\section{The Election of Manganese Patriarch}

\section{Runhong Zhang §, Laiying Zhang *, Yaxian Zhu *}

College of Chemistry and Chemical Engineering, Xiamen University, Xiamen 361005, Fujian Province, P. R. China.

Abstract: Manganese has played an essential role in human life and production. Herein, this paper introduces the manganese's resources, properties and applications. Our aim is to make it easier for readers to understand in a more lively way.

Key Words: Manganese; Property; Application

1774 年的一天, 瑞典著名化学家 C. W. Scheele 将一块矿石(软锰矿)送给朋友 J. G. Gahn, Gahn 将这块矿石与木炭和油等一起加热, 首次制备出金属锰, 从此锰家族正式以 “manganese” 的称号登 上历史舞台 ${ }^{[1]}$ 。

锰家族是个历史悠久的老牌家族, 他们的身影曾出现在旧石器时代晚期洞穴的壁画上, 中国新 石器时代的陶器上, 古埃及法老时代的玻璃制造业中……在现代的钢铁制造、储氢材料、电池制造 等产业中也常常见到他们忙碌的身影。

锰家族的人口众多, 在过渡金属家族圈里, 他们的人口数量仅次于铁家族和钛家族, 占地壳岩 石圈的 $0.085 \%{ }^{[2]}$ 。

家族之中人才辈出, 前辈们尚志在千里, 却也不乏后起之秀。因此, 族长位置的竞争十分激烈。

今天到场参与竞选的是锰家族几个龙头企业的掌权人一锰结核、二氧化锰、高锰酸钾及二价 锰离子。

单质锰宝主持竞选, 他是银灰色的金属, 脾气干脆但是体格不大好。为了以单质的状态多存在 一段时间, 他戴上了氮气面罩 ${ }^{[1]}$ 。锰宝道: “咱们锰家族在元素王国排位 25 , 住在第四周期、VIIB 族。咱们的主业是炼钢, 铁是咱们的老伙计, 他和碳混得风生水起的时候也没忘了我们。约有 $95 \%$ 的锰矿石都用来制造钢, 作为合金金属, 锰可以增加钢的硬度, 谓之高锰钢。同时咱们还有净化剂 
功效, 铁结合硫会变脆, 锰把硫抓住进入炉渣, 可提高钢的性能; 抓住炼钢过程中掺杂的氧气, 可 以避免钢中形成气泡或沙孔 ${ }^{[1]}$ 。”

锰宝顿了一下，“除此之外，我们家族还有很多用途，这都离不开矿产资源。锰结核是近年来 矿产资源的后起之秀, 因此, 我们请锰结核首先发表演说。”

\section{1 锰结核一一锰资源开发}

黑头褐脑、圆溜溜的锰结核走上台, 向大家一拱手：“哎呀, 惭愧！惭愧！我今天是代表着锰 家族的矿产长老们来的。我是矿产界的晚辈, 虽然在地球已生存了几十亿年了, 但 1873 年才和人类 第一次相见。”

“咱们锰家族，是地球上丰度居第 12 位的元素，存在于 300 种不同而又广泛分布的矿物中 ${ }^{[1]}$ 。 说到矿产, 可不能忘本。最初咱们能跟人类打上交道, 登上元素周期表, 是托了软锰矿 $\left(\mathrm{MnO}_{2}\right)$ 长老 的福啊。”

台下的软锰矿捻着胡须微微一笑。锰结核继续说:

“软锰矿是锰矿产中最常见的氧化物和碳酸盐的次生矿床; 而黑锰矿 $\left(\mathrm{Mn}_{3} \mathrm{O}_{4}\right)$ 和菱锰矿 $\left(\mathrm{MnCO}_{3}\right)$ 是由原生的硅酸盐矿床经风化生成。矿床持续风化, 于是锰呀、隔壁老铁和一些其他的金属兄弟的 氧化物胶体颗粒被不断地冲刷入海, 这些颗粒在海水的巨大压力下聚集起来形成锰结核(图 1) ${ }^{[1]}$, 这 便是我的由来了。”
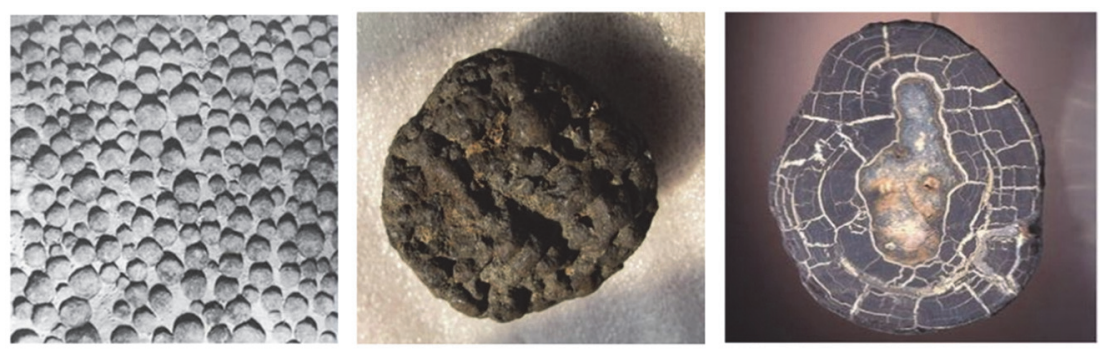

图 1 锰结核

锰结核咳了一声，继续说道，“所以，我实际上是一种海底多金属矿体，平常主要以硅酸盐和 难溶性高锰酸盐的混合物形式存在, 主要成分是锰和铁的氧化物和氢氧化物, 所以大家也可以叫我

“铁锰结核”。有时候我吃得胖, 是个球状物; 有时候我长得像葡萄; 不想装扮的时候我就以炉渣 状在外行走。别看我现在有几十千克重, 最小的锰结核宝宝们, 才几微米大呢！[3]”

“在诞生之初, 我们就有招兵买马的念头, 把海水或海底沉积物间隙水的成矿物质们召集到一 起, 于是, 我们也吸收一些钴、镍、铜等二价阳离子弟兄, 人多力量大啉。而且一片海域长大的锰

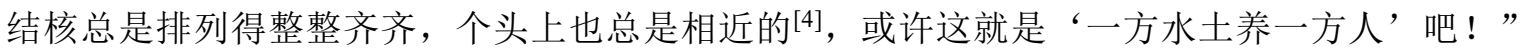

“虽然我这个核里含锰量大概只有百分之十几到百分之二十几，比当前商业开采的要求 $35 \%$ 要 低, 但是得益于我在生长过程中还会吸引一些铜、钴、镍等具有经济价值的元素, 如果联合回收这 些元素, 那经济收益还是相当可观的 ${ }^{[4]}$ 。居住在太平洋的兄弟们, 身体里锰、镍、铜的总量, 就是大 陆上同种元素家族的上百倍。我们锰结核虽然单个拎出来个头不大, 但是核多力量大, 总量可达三 万多亿吨哦, 而且我们还在不断地缓慢长大呢。”

别看锰结核䄓态可掬, 说起话来绝不含糊: “数量大, 又可持续, 许多冶金企业相当看好我们。 20 世纪 80 年代的统计数据显示, 当时已经有 100 多家企业从事锰结核勘探的相关工作啦 ${ }^{[3]}$, 我们 是锰家族资源的未来之星。”

“但是，如何从深海把大大小小的锰结核开发出来呢？”台下有人小声嘟噮着。 
“请上道具。”锰结核一挥手, 亮出了两组模拟道具: 水车和吸尘器。

只见锰宝宝们迫不及待地钻入水车的各个桶里, 像玩摩天轮一样开心活跃起来。

“大家请看, 目前开发锰结核的方法主要有三种, 分别是链斗法、空气升举法和水力升举法。”

“这些方法原理跟人类生活中的工具一一水车和吸尘器非常的类似。链斗法的工具是串联起来 的传输链, 利用转动力, 就像水流带动的老水车一样, 可以不停地把海底的锰结核带到工作船上来。 而空气升举法和水力升举法则是利用高压气或水力, 将水、泥同锰结核一同吸上来, 后期再进行处 理分离 $[3]$ 。有意向的朋友们欢迎加盟哦！

那边有工作人员一路小跑上台, 在锰结核身旁附耳道: “您串场啦! 今天咱们是竞选族长专场, 拉投资的事在下一场呢！”

\section{2 二氧化锰一工业起始原料代表}

锰结核慢悠悠踱步离开了, 一个褐色金属宝宝走上台。有人调㑆道: “锰宝, 哦不, 现在该叫 你黑宝了，你怎么把面罩摘了？”

“接下来要上台竞选演说的, 是我这外衣的堂兄—二氧化锰。” 锰宝扮了个鬼脸, “我摘下面 罩, 换上氧化衣, 为了应景啊。”

二氧化锰在锰家族里举足轻重, 不过话不多, 性子不活泼。具有金红石结构的 $\beta$-二氧化锰, 作 为唯一符合软锰矿化学计量式的晶型代表二氧化锰们上台, 他向大家微微领首 ${ }^{[1]}$ 。

“大家好! 我们二氧化锰, 是多胞胎, 晶型结构复杂, 存在 5 种主晶及 30 多种次晶 ${ }^{[5]}$, 我是 $\beta$ 晶型, 较为常见的还有 $\alpha 、 \gamma$ 和 $\delta$ 晶型。”

“锰家族的主业是炼钢, 在这个领域二氧化锰主要是作为原料来使用, 在这里就不再赘述。在 治金以外的领域, 我们也大有可为。其中最大的非冶金应用是制造干电池, 我们在干电池里作为去 极化剂, 管控好溶液中的氢离子不要抢走电子, 变成氢气逸散而带来的电池效率降低。”

$\beta$-二氧化锰润了润嗓子: “下面, 介绍一下我们其他比较突出的贡献——超级电容器电极材料、 空气电极催化剂、纳米片改性薄膜以及软磁材料锰锌铁氧体。”

二氧化锰显然是有备而来。由于力求完美的个性, 他还为大家准备了 PPT。

“1999 年, Goodenough 老爷子, 他可是在 2019 年获得了诺贝尔化学奖的哦, 首先发现了 $\mathrm{MnO}_{2}$ 的噟电容行为。因为我们劳动力众多且低廉, 竞争力高, 在超级电容器领域迅速抢占市场 ${ }^{[5]}$ 。”

他说着点开了一个游戏, 场景可供选择, 他说道: “晶型、比表面积、表面形态等因素都会影响 $\mathrm{MnO}_{2}$ 的电容性能, 请玩家们在 $\alpha 、 \beta 、 \gamma 、 \delta$ 二氧化锰里挑选自己的角色吧。”

年轻的观众走上台, 有玩家眼疾手快地抢了 $\alpha-\mathrm{MnO}_{2}$, 果然因为比表面积和孔隙率更大, 在电极 比容量 PK 时打败了其余三位玩家 ${ }^{[5]}$ 。

$\beta$-二氧化锰的小粉丝悄悄提示选择他的玩家: “你跟他比比倍率性能和稳定性, 你能赢的!”

台下响起抗议声, $\beta$-二氧化锰严厉抨击了小粉丝, 并主动退出游戏。

只剩下 $\alpha 、 \gamma 、 \delta$ 三位, 游戏继续进行, $\alpha$ 玩家在结构稳定性中惜败。不过, 随后又以高出一等的 放电电压拔得空气电极催化剂竞赛的头筹 ${ }^{[6]}$, 扳回一城。

接下来是改性薄膜游戏, 在这里角色们都是同样的二氧化锰了, 共同对付造成 “穿梭效应”、导 致锂硫电池容量在使用过程中不断衰减的中间产物多硫离子们。大家在电池里玩起矿工游戏, 操纵 着纳米片改性薄膜中的二氧化锰吸附住多硫离子们, 再将它们催化转化, 评分标准有三一一锂硫电 池的容量、倍率性能和循环稳定性 ${ }^{[7]}$ 。大家玩得都很尽兴。

“不知道我们锰家族中的其他化合物是否想发展上述业务? 我倒是可以为大家牵线搭桥。”

突然, 电话铃声大作，二氧化锰向大家拱手道：“抱慊！公司有急事，我先走一步。”

看到二氧化锰乘车离开的背影, 有人小声感慨: “不愧是锰家族的发展之星呀, 都换上混合动力 车了。” 
锰宝道: “这车上的电子设备, 大多是软磁铁氧体制成的 ${ }^{[8]}$, 这也是二氧化锰旗下的产业链之一 哪!”

一时间，二氧化锰当选族长的呼声很高。

\section{3 高锰酸钾一一氧化小能手}

“什么? 高锰酸钾改变原定计划, 要给大家露一手? ”

锰宝飞速地组织了一段主持词: “在过渡元素家族, 咱们锰家族是氧化态变化最多的元素之一, 可以 $+2 、+3 、+4 、+6 、+7$, 颜色变化也丰富多彩。今天高锰酸钾要为大家带来即兴表演一一氧化态 大变脸, 大家掌声欢迎! ”

只见烧杯舞台旋转起来, 一队高锰酸钾青年分为三组, 分别进入 $\mathrm{NaOH}$ 溶液、 $\mathrm{H}_{2} \mathrm{O}$ 溶液与硫酸 溶液。 $\mathrm{MnO}_{4}^{-}$与 $\mathrm{K}^{+}$分离, 开始自在地游动, 此时三个舞台的溶液都是紫红色的; 亚硫酸钠从烧杯上 方注入, 碱性舞台的 $\mathrm{MnO}_{4}^{-}$小哥哥们把脸一抹, 变成了墨绿色的 $\mathrm{MnO}_{4}^{2-}$; 中性舞台里的 $\mathrm{MnO}_{4}^{-}$沉降到水 底, 成为棕色的 $\mathrm{MnO}_{2}$; 酸性舞台的紫色慢慢裉去, $\mathrm{MnO}_{4}^{-}$们不见了吗? 原来他们变成了肉色的 $\mathrm{Mn}^{2+}$, 让自己隐身啦!

硫酸先生当起了指挥棒, 一滴滴指引着 $\mathrm{pH}$ 降低, 碱性舞台悄悄变了模样: $\mathrm{MnO}_{4}^{2-}$ 们有的摇曳在 溶液中, 又换回了紫色的衣裳, 变回 $\mathrm{MnO}_{4}$; 有的让自己沉降到水底, 成为棕色的 $\mathrm{MnO}_{2}$, 沉淀的 $\mathrm{MnO}_{2}$ 兴奋地拉上中性舞台的同伴, 浓盐酸先生游过来, 帮助他们打造出梦幻的黄绿色舞台, 肉色的 $\mathrm{Mn}^{2+}$ 在烟雾中翩翩而来。

变脸总要结束, $\mathrm{MnO}_{4}^{-}$还要回来演讲啊。硫酸指挥棒拍拍脑袋, 拉上 $\mathrm{NaBiO}_{3}$ 来助阵, $\mathrm{Mn}^{2+}$ 又恢 复了 $\mathrm{MnO}_{4}^{-}$的模样 ${ }^{[9]}$ 。

台下的观众都看痴了。

“大家好, 我是高锰酸钾。我有“两性”: 强氧化性和不稳定性。”高锰酸钾很绅士地说道。

“由于强氧化性，特别在酸性条件下氧化性强，可以氧化大部分有机物和一些金属离子，我经 常被用于医学上的消菌杀毒、环境水污染治理剂, 在园林方面, 我还可以防治根腐病等花卉病。由 于反应定量、快速, 高锰酸钾滴定法已经使用很多年了。”

“至于不稳定性么, 大家最熟悉的或许是我在实验室制备氧气的功效了, 加热就可以使我分解 产生锰酸钾、二氧化锰和氧气。因此, 大家一定要记住, 高锰酸钾溶液一定要随配随用, 不然我可 是会失活的哦。不过, 我还是个污染物, 用的太多、浓度太高会增加生态风险。”

气氛轻松愉快, 人群中传出低低的笑声。

\section{4 二价锰一一宣传大使}

$\mathrm{Mn}^{2+}$ 是锰最稳定的氧化态之一, 她也没有叫上别的元素助阵, 独自上台, 为大家介绍二价锰在 光催化方面的应用。

“光催化是个美妙的反应，过渡金属掺杂到光催化材料中，可以帮助提高光催化性能。因为, 咱们过渡金属离子结交的光波多, 吸光范围更广, 光更愿意把礼物一一能量带给我们, 我们跟反应 物要好, 可以轻松将他们叫到催化材料旁拉住他们的手说话; 同时咱们化合价多, 喜欢和其他元素 朋友们交换电子, 可以在催化剂表面产生缺陷或改变材料结晶度, 降低电子与空穴的复合效率, 提 高光催化性能。”

“比如我一 $-\mathrm{Mn}^{2+}$ 掺杂进二氧化钛, 就能够促进光催化反应的进行。当然在座的锰家族的其他 化合物, 如二氧化锰、隐钾锰矿等, 也具有很好的光催化活性 ${ }^{[10]}$ 。”

“除了工业领域, 我们镇还在人类的身体里, 是人体必需微量元素之一, 含量低但是力量大, 参与人体骨骼、脑等组织的构成, 主要分布在人类的脑垂体中。同时, 也是人体内金属酶和激活酶 的重要组成成分, 假如元素界举办元素与人类健康大赛, 咱们锰是一定要参加的。遗传、免疫、代 
谢, 咱们可以说是样样参与。例如, 一定浓度范围内的游离的我可以作为核酶的激动剂 ${ }^{[11]}$, 台下的 $\mathrm{Mn}(\mathrm{III})$ 是可以保护人类参与呼吸的重要细胞器线粒体膜的锰超氧化物歧化酶的活性中心 ${ }^{[12]}$, 在人体 许多生命活动如尿素合成、糖原异生、胆固醇代谢中都有我们的身影。若是人体中缺少锰元素, 会 出现皮炎、骨质疏松、关节疾病等症状。不过好在需求量小, 简单来说, 人类只需正常饮食就可以 和我们建立良好的友谊关系 ${ }^{[13]}$ 。”

“但是大家可千万不要一股脑地和一个人交朋友，摄入过多锰对人类可不是什么好事情。环境 中若锰含量较高, 饮用锰含量较高的水, 都会对人体产生一定的危害。长期吸入含低浓度锰的气体 可引起慢性锰中毒, 出现如神经衰弱综合征及植物神经功能障碍; 误服锰化合物则会引起胃肠道症 状, 严重者甚至会休克而死亡。而且更要注意的是, 化合价低的与溶解度大的锰化合物通常要比溶 解度小的锰化合物的毒性更大 ${ }^{[14]}$ 。因此, 咱们也要提醒人类朋友们注意, 虽然我们是好朋友, 交往 间也要保持适度的距离, 距离产生美嘛!”

台下响起了热烈的掌声。

锰宝笑着走上台: “大家心里可有适合的族长人选了？”

有说锰结核是未来之星的, 有说软锰矿德高望重的, 有说高锰酸钾价态高却脚踏实地的, 还有 说二价锰离子热心环保、关注民生的……

硫酸锰举起了手: “这可不好办呀, 分工都不一样, 不好比呢。”

在场的化合物们也纷纷表示赞同。

你能帮锰家族解决这个难题吗?

[1] 格林伍德(Greenwood, N. N.) (英), 厄恩肖(Earnshaw, A.) (英). 元素化学(下册). 曹庭礼, 夏鲁惠, 耿承延, 等译. 北京: 高等教育出版社, 1996: 196-209

[2] 张兆麟. 化学教育, $\mathbf{1 9 9 5}$, No. $10,1$.

[3] 周怀阳. 自然杂志, 2015, 37 (6), 397.

[4] 孟迪. 管理观察, 2019, No. 26, 80.

[5] 危震坤, 华小珍, 肖可, 周贤良, 叶志国. 电化学, 2015, 21 (4), 393.

[6] 冯攀, 俞小花, 李永刚, 俞双林, 李荣兴, 谢刚. 材料科学与工程学报, 2019, 37 (3), 368 .

[7] 彭娜, 翟鹏飞, 王景涛, 王俊晓, 刘咏. 化工学报, 2020, No. 5, 2389.

[8] 李超群, 田宗平, 曹健, 周永兴, 邓圣为, 陈铮. 中国锰业, 2016, 34 (6), 91.

[9] 刘新锦, 朱亚先, 高飞. 无机元素化学. 第 2 版. 北京: 科学出版社, 2010: 234-238.

[10] 张立艳, 张英锋, 马子川. 化学教育, 2011, No. 1, 4 .

[11］李淑芬, 张宏. 工企医刊, 1999, No. 2, 106.

[12] 梅光泉, 应惠芳. 宝鸡文理学院学报(自然科学版), 2004, No. 1, 29.

[13] 陈霞飞. 质量与标准化, 2015, No. 5, 26.

[14] 魏少征. 化工劳动保护(工业卫生与职业病分册), 1991, No. 4, 190. 